\title{
Thermodynamics of carbon point defects in hexagonal boron nitride
}

\author{
Marek Maciaszek $\odot,{ }^{1,2, *}$ Lukas Razinkovas $\odot,{ }^{2}$ and Audrius Alkauskas $\odot^{2,3, \dagger}$ \\ ${ }^{1}$ Faculty of Physics, Warsaw University of Technology, Koszykowa 75, 00-662 Warsaw, Poland \\ ${ }^{2}$ Center for Physical Sciences and Technology (FTMC), Vilnius LT-10257, Lithuania \\ ${ }^{3}$ Department of Physics, Kaunas University of Technology, Kaunas LT-51368, Lithuania
}

(Received 3 November 2021; accepted 21 December 2021; published 21 January 2022)

\begin{abstract}
We present a first-principles computational study of the thermodynamics of carbon defects in hexagonal boron nitride (hBN). The defects considered are carbon monomers, dimers, trimers, and larger carbon clusters, as well as complexes of carbon with vacancies, antisites, and substitutional oxygen. Our calculations show that monomers $\left(\mathrm{C}_{\mathrm{B}}, \mathrm{C}_{\mathrm{N}}\right)$, dimers, trimers, and $\mathrm{C}_{\mathrm{N}} \mathrm{O}_{\mathrm{N}}$ pairs are the most prevalent species under most growth conditions. Compared with these defects, larger carbon clusters, as well as complexes of carbon with vacancies and antisites, occur at much smaller concentrations. Our results are discussed in view of the relevance of carbon defects in single-photon emission in hBN.
\end{abstract}

DOI: 10.1103/PhysRevMaterials.6.014005

\section{INTRODUCTION}

Over the past two decades, hexagonal boron nitride (hBN) has gained significant interest both as an insulator used in conjunction with other layered two-dimensional (2D) materials [1] and as an active optical medium itself [2]. The large band gap of hBN, $6.08 \mathrm{eV}$ [3], makes it unique among the layered compounds. For all potential applications of hBN, whether as a passive insulator or as an active optoelectronic material, the purity of single crystals or epitaxial layers is crucial. Unfortunately, the growth of ultrapure material has been challenging [4]. In part, this is because the defect chemistry of boron nitride is still poorly understood.

Due to an intermediate position in the periodic table between boron and nitrogen and a similar crystal structure, carbon plays an exceptional role in hBN. It is known that carbon is often present as an unintentional impurity in hBN grown by a variety of different methods. Experiments on bulk hBN samples produced by high-pressure high-temperature synthesis with barium boron nitride (Ba-BN) as a solvent [5], a frequently employed growth method, showed the existence of carbon-rich domains [6]. These domains extended both along the $c$ axis as well as in the 2D layers, and the density of carbon atoms in these domains exceeded $10^{18} \mathrm{~cm}^{-3}$ [6], as indicated by secondary-ion mass spectroscopy. The presence of carbon in $\mathrm{hBN}$ was also revealed by direct imaging via annular dark-field electron microscopy [7] on samples exfoliated from bulk hBN.

\footnotetext{
*marek.maciaszek@pw.edu.pl

†audrius.alkauskas@ftmc.lt
}

Published by the American Physical Society under the terms of the Creative Commons Attribution 4.0 International license. Further distribution of this work must maintain attribution to the author(s) and the published article's title, journal citation, and DOI.
The interest in point defects in $\mathrm{hBN}$ in general and carbonrelated defects in particular has recently increased due to the observation of single-photon emission in this material [8-11]. The emission is believed to originate at point defects; for an overview of this fast-developing field, we refer the reader to Ref. [12]. Compared with materials such as diamond or silicon carbide, a two-dimensional material such as hBN offers certain advantages as a host of quantum emitters, e.g., the possibility of high photon extraction efficiency. The reports about single-photon emitters (SPEs) in hBN are numerous. Most of the emitters have structured luminescence bands with zero-phonon line (ZPL) energies in the range 1.6-2.1 eV [8-11], accompanied by a clearly pronounced phonon sideband. In Ref. [13], Mendelson et al. used various experimental techniques and samples grown by several methods to show that single-photon emission in this energy range is carbon related (with most SPEs having ZPL energies at about $2.1 \mathrm{eV}$ ). This means that either emitters directly contain carbon atoms or carbon influences the formation of other defects. Several recent theoretical works suggested carbon-related defects to explain emission in this energy range. In particular, Sajid and Thygesen proposed the complex of a substitutional carbon with a nitrogen vacancy, $\mathrm{C}_{\mathrm{B}} V_{\mathrm{N}}$, as one of the candidates [14]. Jara et al. [15] suggested that carbon trimers $C_{3}\left(C_{2} C_{B}\right.$ and $\mathrm{C}_{2} \mathrm{C}_{\mathrm{N}}$ ) have optical signatures resembling some of the emitters in the 1.6-2.1-eV range, also in agreement with the involvement of carbon in single-photon emission. The hypothesis about the $\mathrm{C}_{2} \mathrm{C}_{\mathrm{N}}$ trimer, in particular, has been recently backed by Li et al. [16]. Several calculated optical properties of this defect matched the experimental observations well. Finally, Auburger and Gali performed calculations of optical excitation energies and magnetic resonance signatures of several carbon-containing defects. They concluded that carbon on the boron site $C_{B}$ is the most likely candidate to explain the emission [17].

Apart from quantum emission in the red and the infrared (IR) spectral regions, in Ref. [9], single-photon emission in 
the near-ultraviolet (near-UV) range has been reported. The optical signal of the emitter is nearly identical to the wellknown 4.1-eV luminescence line in hBN $[18,19]$. It has been known for some time that deliberate doping of $\mathrm{hBN}$ with carbon increases the intensity of this line [18,20,21]. Therefore the 4.1-eV line, frequently observed in nominally undoped samples [22], has often been used as a proxy for carbon in hBN. Historically, the $4.1-\mathrm{eV}$ line was often attributed to carbon on the nitrogen site [23]. However, it was recently shown that $\mathrm{C}_{\mathrm{N}}$ is not the origin of the $4.1-\mathrm{eV}$ luminescence [24]. Instead, we have recently provided theoretical evidence that the origin of this line is the carbon dimer defect $\mathrm{C}_{2}$ [25]. The $\mathrm{C}_{\mathrm{N}} \mathrm{O}_{\mathrm{N}}$ pair has also been suggested to explain the 4.1-eV luminescence [26]. However, a stoichiometric StoneWales defect that does not involve carbon at all has also been recently proposed [27]. All three of these defects are expected to emit in the UV range, but the debate regarding the exact chemical nature of the $4.1-\mathrm{eV}$ emitters seems to continue.

It is clear that present evidence strongly suggests the importance of carbon-related defects in optical emission in $\mathrm{hBN}$, as carbon appears to be an essential component of SPEs in both the red-IR and UV regions. However, it can also be concluded that a universal consensus regarding an assignment of a specific defect to a particular luminescence line has not yet been reached, despite intensive experimental and theoretical investigations. This situation is certainly unsatisfactory, as it hinders the development of hBN devices based on color centers. Most of the theoretical investigations that suggested a certain defect giving rise to a specific luminescence band analyzed one or at most a few defect candidates. However, a broader picture of carbon defects in $\mathrm{hBN}$ has been missing.

In this paper, we present first-principles density functional theory calculations of carbon in hBN. In particular, we study single carbon substitutionals, carbon dimers, trimers, non-nearest-neighbor carbon pairs, and larger carbon clusters, as well as complexes of substitutional carbon with vacancies, antisites, and substitutional oxygen. Our goal is to analyze the thermodynamics of carbon-related species under various growth conditions. These results provide a general picture of carbon-related defect chemistry that will be helpful for interpreting existing data and designing new experiments.

For the sake of completeness, we must state that a very different class of single-photon emitters in $\mathrm{hBN}$ has been recently discovered. These emitters feature broad bands peaking at about $830 \mathrm{~nm}(\sim 1.5 \mathrm{eV})$. They are attributed to negatively charged boron vacancies $[28,29]$. As of now, these are the only color centers in $\mathrm{hBN}$ where a consensus [30] regarding their chemical structure exists.

The paper is organized as follows. In Sec. II, the theoretical approach is outlined. In Sec. III, we discuss defects analyzed in this paper. Calculated formation energies and equilibrium defect concentrations are analyzed in Sec. IV. The relevance of our calculations for single-photon emission experiments is discussed in Sec. V. In Sec. VI, we analyze the range of validity of the formation energy formalism for carbon-related defects in $\mathrm{hBN}$ and present the results for solubility limits of carbon in hBN. Section VII concludes our paper.

\section{THEORETICAL METHODOLOGY}

Our first-principles calculations were performed using the Heyd-Scuseria-Ernzerhof [31] hybrid functional based on screened Fock exchange. The screening parameter was $0.2 \AA^{-1}$, and a mixing parameter, which indicates the fraction of the screened Fock exchange admixed to the local exchange, was set to $a=0.31$. The local exchange is described via a modified Perdew-Burke-Ernerzof density functional [32]. The mixing parameter was chosen to obtain a good description of the band gap. The plane-wave basis set with the kinetic energy cutoff $500 \mathrm{eV}$ was used, and the interaction of electrons and ions was described via the projector-augmented wave approach [33]. Positions of ions were relaxed until all forces fell below $0.01 \mathrm{eV} / \AA$. We used orthorhombic supercells with 240 atoms to model defects. The lattice vectors of supercells are $5(\mathbf{a}+\mathbf{b}), 3(\mathbf{a}-\mathbf{b}), 2 \mathbf{c}$, where $\mathbf{a}, \mathbf{b}$, and $\mathbf{c}$ are primitive vectors of the Bravais lattice. To take into account van der Waals interactions between the $\mathrm{hBN}$ layers, we applied the GrimmeD3 scheme [34]. Geometry relaxation was performed and total energies were computed including these van der Waals corrections. A single $\Gamma$ point was used for the Brillouin zone integration. Calculations have been performed using the Vienna Ab Initio Simulation Package (VASP) [35]. The described methodology reproduces experimental parameters very well. The calculated band gap is $5.91 \mathrm{eV}$ (experimental value $6.08 \mathrm{eV}$ [3]), the formation enthalpy of the compound equals $-2.89 \mathrm{eV}$ per formula unit (experimental value $-2.60 \mathrm{eV}$ [36]), and lattice constants are $a=2.49 \AA$ and $c=6.55 \AA$ (experimental values 2.50 and $6.65 \AA$, respectively [37]).

Let us consider a point defect in a charge state $q$. Let $n_{\mathrm{C}}$, $n_{\mathrm{B}}$, and $n_{\mathrm{N}}$ be the number of carbon, boron, and nitrogen atoms added to the $\mathrm{hBN}$ supercell when the defect is created ( $n_{\mathrm{B}}$ and $n_{\mathrm{N}}$ can be negative, meaning that atoms have been removed). The formation energy of the defect as a function of the electron chemical potential (or Fermi level) $E_{F}$ is $[38,39]$

$$
\begin{aligned}
\Delta H_{f}(D, q)= & E_{\text {tot }}(D, q)-E_{\text {bulk }}-n_{\mathrm{C}} \mu_{\mathrm{C}}-n_{\mathrm{B}} \mu_{\mathrm{B}}-n_{\mathrm{N}} \mu_{\mathrm{N}} \\
& +q\left(E_{V}+E_{F}\right)+E_{\text {corr }} .
\end{aligned}
$$

Here, $q$ is the charge state of the defect, $E_{\text {tot }}(D, q)$ is the total energy of the supercell containing one defect, $E_{\text {bulk }}$ is the total energy of the defect-free supercell. $\mu_{\mathrm{B}}, \mu_{\mathrm{N}}$, and $\mu_{\mathrm{C}}$ are chemical potentials of boron, nitrogen, and carbon, respectively. $E_{V}$ is the energy of the valence band maximum (VBM), with respect to which $E_{F}$ is referenced. $E_{\text {corr }}$ is a correction term that accounts for spurious interactions of charged defects in periodic supercells, and we use the form of the correction provided in Ref. [40]. The value of the Fermi energy for which charge states $q$ and $q^{\prime}$ have equal formation energies is called the charge-state transition level and is labeled $\left(q / q^{\prime}\right)$ [39].

Atomic chemical potentials that appear in Eq. (1) reflect the chemical environment during growth. The equilibrium nature of growth of $\mathrm{hBN}$ is ensured by the condition $\mu_{\mathrm{B}}+\mu_{\mathrm{N}}=$ $\mu_{\mathrm{BN}}$, where $\mu_{\mathrm{BN}}$ is the total energy of bulk $\mathrm{hBN}$ per one formula unit. Within the constraint of equilibrium growth, $\mathrm{N}$-rich (B-poor) conditions correspond to the value of $\mu_{\mathrm{N}}=1 / 2 \mu_{N_{2}}$, where $\mu_{N_{2}}$ is the energy of the $\mathrm{N}_{2}$ molecule. Under B-rich (N-poor) conditions, $\mu_{\mathrm{B}}=\mu_{\mathrm{B}_{\text {bulk }}}$, where $\mu_{\mathrm{B}_{\text {bulk }}}$ is the total energy of bulk boron per one atom. The actual chemical potentials must always be within these two limits. Therefore 
we discuss formation energies for both N-rich and N-poor conditions. The chemical potential of carbon $\mu_{\mathrm{C}}$ is equal to the energy of carbon in bulk graphite. This is the carbon-rich limit, which will be discussed in more detail in Secs. V and VI.

Apart from carbon, it is known that oxygen is another prevalent impurity in $\mathrm{hBN}$ [7]. Therefore we choose to model the carbon chemistry in two situations: without and with oxygen. The formation energy of oxygen-containing defects is calculated using an expression similar to Eq. (1), where oxygen atoms are accounted for via the oxygen chemical potential $\mu_{\mathrm{O}}$. We consider the oxygen-rich limit. Due to a very low formation enthalpy (high stability) of $\mathrm{B}_{2} \mathrm{O}_{3}$, the maximum chemical potential of oxygen is determined by the energy of $\mathrm{O}$ in $\mathrm{B}_{2} \mathrm{O}_{3}$ under both $\mathrm{N}$-rich and $\mathrm{N}$-poor conditions. The need to consider the formation of $\mathrm{B}_{2} \mathrm{O}_{3}$ when determining the oxygen chemical potential brings an additional complication. Despite the low formation enthalpy of $\mathrm{B}_{2} \mathrm{O}_{3}$, its melting temperature is $\sim 750 \mathrm{~K}$, below typical growth temperatures of hBN $(T>$ $1000 \mathrm{~K})$. In this paper, we do not consider the liquid phase and take the limiting value of $\mu_{\mathrm{O}}$ by considering solid $\mathrm{B}_{2} \mathrm{O}_{3}$. This makes our calculations for O-containing defects slightly more qualitative.

Once the formation energies of defects are known, their equilibrium concentrations are determined using thermodynamical simulations $[41,42]$. In brief, the concentration of a certain defect $D$ in a charge state $q$ at temperature $T$ is determined via its formation energy:

$$
N_{D, q}(T)=N_{D, \text { sites }} e^{-\Delta H_{f}(D, q) / k_{B} T},
$$

where $N_{D \text {,sites }}$ is the density of sites for the defect to form (we use the terms "density" and "concentration" as synonyms). We express $N_{D \text {, sites }}$ via $N_{D \text {, sites }}=g_{D} N_{\text {sites }}$, where $N_{\text {sites }}$ is the density of atomic sites in hBN. This definition explains the physical meaning of the factor $g_{D}$. In the literature, $g_{D}$ is sometimes called the lattice multiplicity factor or simply the degeneracy factor. For example, $g_{D}=1 / 2$ for single substitutional defects in hBN, $g_{D}=3 / 2$ for carbon dimers, $g_{D}=6$ for carbon tetramers (cis and trans conformers have nearly the same energies, see below), $g_{D}=1 / 2$ for closed-ring carbon hexamers, etc. (for a description of different carbon defects, see Sec. III). The position of the Fermi level $E_{F}$ is determined by imposing the charge-neutrality condition $\sum_{D, q} N_{D, q} q=0$ $[41,42]$. The sum is carried over all defects included in the modeling. Due to a large band gap of hBN, we exclude free carriers from our simulations. We verified that including them does not affect the main results of the paper. Equation (2) for all considered defects and the charge-neutrality condition form a set of equations that are solved self-consistently.

The outlined methodology has several shortcomings that need to be discussed. First, we state at the outset that we model only the thermodynamics of carbon-related defects. In reality, kinetic effects (e.g., diffusion) could lead to defect concentrations being different from those predicted by thermodynamics. Due to the complexity of the problem, we abstain from discussing kinetic effects in this paper. Instead, our goal is to establish the limits set by equilibrium thermodynamics. The second shortcoming is related to vibrational contributions to free energies. More rigorously, one should determine the formation enthalpy at finite temperatures. This

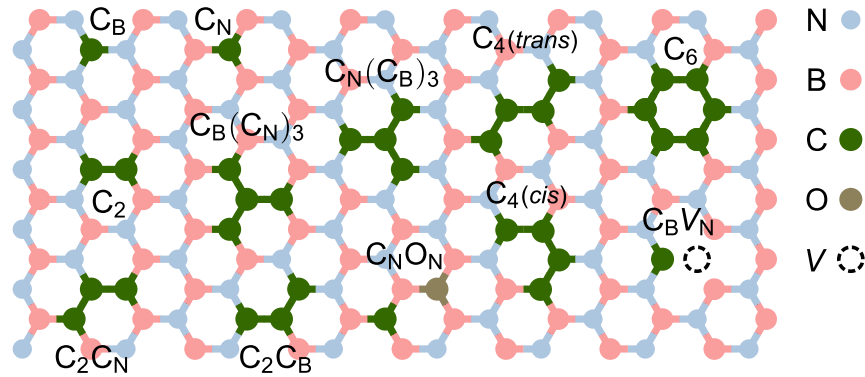

FIG. 1. A subset of carbon-related defects considered in this paper: single substitutional defects $C_{B}$ and $C_{N}$, carbon dimer $C_{2}$, carbon trimers $\mathrm{C}_{2} \mathrm{C}_{\mathrm{N}}$ and $\mathrm{C}_{2} \mathrm{C}_{\mathrm{B}}$, carbon tetramer $\mathrm{C}_{4}$ (both cis and trans configurations are shown), triangular starlike defects $\mathrm{C}_{\mathrm{N}}\left(\mathrm{C}_{\mathrm{B}}\right)_{3}$ and $\mathrm{C}_{\mathrm{B}}\left(\mathrm{C}_{\mathrm{N}}\right)_{3}$, hexamer $\mathrm{C}_{6}$, the $\mathrm{C}_{\mathrm{B}} V_{\mathrm{N}}$ complex, and the $\mathrm{C}_{\mathrm{N}} \mathrm{O}_{\mathrm{N}}$ pair.

means that the vibrational contribution to free energies should be included in Eq. (1) [39]. In this paper, we approximate these energies by their zero-temperature counterparts, as is often done in defect simulations [39].

\section{CARBON DEFECTS}

We include a large number of carbon defects in our analysis. These can be grouped into several distinct sets. (i) Carbon substitutionals and their clusters: monomers $C_{B}$ and $C_{N}$, the dimer $\mathrm{C}_{2}$ (where the two carbon atoms are on adjacent sites), carbon pairs $C_{B}-C_{N}$ (whereby the two carbon atoms are not adjacent), trimers $\mathrm{C}_{3}\left(\mathrm{C}_{2} \mathrm{C}_{\mathrm{N}}\right.$ and $\left.\mathrm{C}_{2} \mathrm{C}_{\mathrm{B}}\right)$, triangular (starlike) defects $C_{N}\left(C_{B}\right)_{3}$ and $C_{B}\left(C_{N}\right)_{3}$, and larger clusters, in particular, $\mathrm{C}_{4}, \mathrm{C}_{5}, \mathrm{C}_{6}, \mathrm{C}_{8}$, and $\mathrm{C}_{10}$. (ii) Complexes of carbon substitutionals with vacancies: $\mathrm{C}_{\mathrm{B}} V_{\mathrm{B}}$ and $\mathrm{C}_{\mathrm{N}} V_{\mathrm{N}}$ (which can be thought of as donor-acceptor pairs), $\mathrm{C}_{\mathrm{B}} V_{\mathrm{N}}$ (donor-donor pair), and $\mathrm{C}_{\mathrm{N}} V_{\mathrm{B}}$ (acceptor-acceptor pair). (iii) Antisite-carbon pairs: $N_{B} C_{B}, N_{B} C_{N}, B_{N} C_{B}$, and $B_{N} C_{N}$. As is clear from the list, we consider both first-neighbor and second-neighbor complexes. Some of the studied defects are shown in Fig. 1.

We exclude carbon interstitials and complexes of interstitials with other defects from our analysis. A few such defects were recently considered in Ref. [43]. They typically have very large formation energies. In addition, due to a layered structure of hBN, interstitials have very low diffusion barriers and thus should be easily annealed [24].

Formation energies of charged defects depend on the Fermi level, which is governed by charge states of all point defects and impurities in the system. As a result, our thermodynamic modeling includes all other defects that might affect the position of the Fermi level. These defects are bare vacancies $V_{\mathrm{B}}$ and $V_{\mathrm{N}}$, as well as antisite defects $\mathrm{N}_{\mathrm{B}}$ and $\mathrm{B}_{\mathrm{N}}$. These are the intrinsic defects with lowest formation energies [24].

When oxygen is present, in addition to the defects discussed above, we also include the oxygen-carbon pair $\mathrm{C}_{\mathrm{N}} \mathrm{O}_{\mathrm{N}}$ (shown in Fig. 1), the substitutional oxygen $\mathrm{O}_{\mathrm{N}}$, and the boron-vacancy-oxygen complex $V_{\mathrm{B}} \mathrm{O}_{\mathrm{N}}$ in our analysis.

\section{FORMATION ENERGIES AND DENSITIES}

In this section, we explicitly discuss only defects that can occur at concentrations $N_{D}>10^{14} \mathrm{~cm}^{-3}$ for growth temperatures $T<2000 \mathrm{~K}$. The reasoning behind this criterion will be 
(a) N-poor

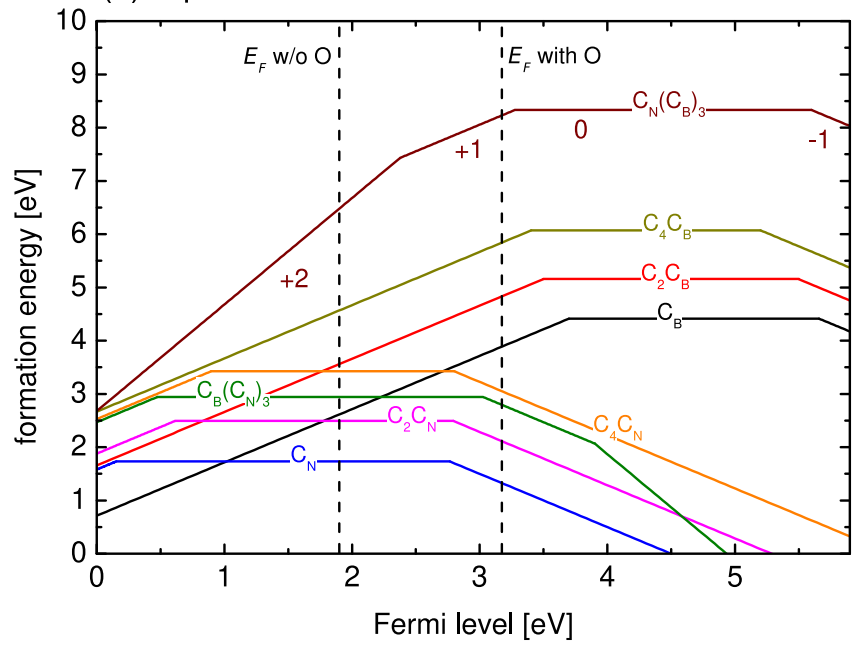

(b) N-rich

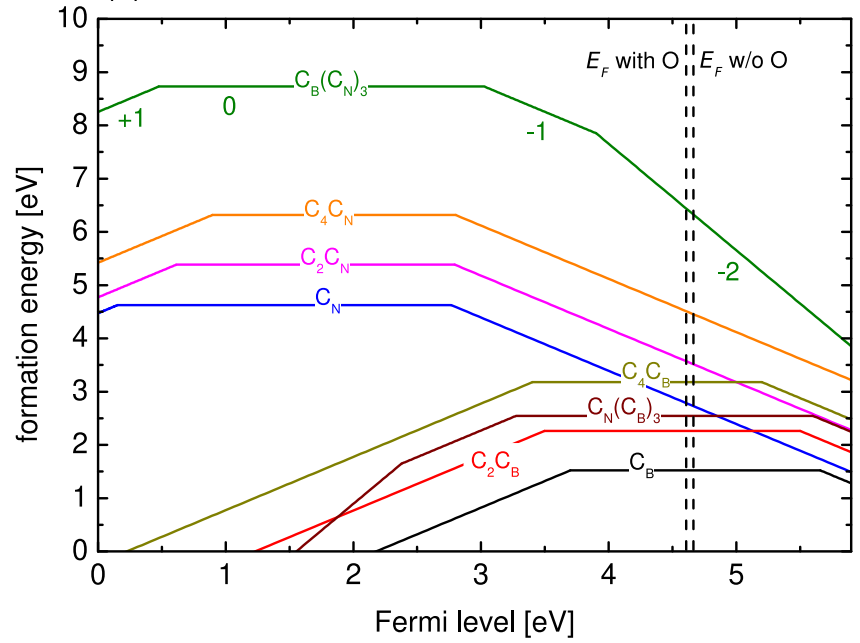

FIG. 2. Formation energies of odd carbon clusters as a function of the Fermi level. Defects considered are single substitutional carbon atoms, carbon trimers, pentamers, and triangular (starlike) defects $C_{N}\left(C_{B}\right)_{3}$ and $C_{B}\left(C_{N}\right)_{3}$. (a) N-poor conditions; (b) N-rich conditions. Vertical dashed lines show the values of our calculated Fermi level with and without (w/o) oxygen. Numbers close to the formation energy line indicate the charge state. For simplicity, the charge state is shown only for one defect. The charge states for other defects can be inferred from the slope of formation energy lines.

discussed in Sec. V. The data for all other carbon defects can be found in the Supplemental Material [44].

\section{A. Formation energies}

Odd carbon clusters. Simple carbon substitutionals $\mathrm{C}_{\mathrm{B}}$ and $\mathrm{C}_{\mathrm{N}}$ are carbon defects that have been investigated previously $[17,24,25,45,46]$. Since a single boron or nitrogen atom is replaced by an impurity carbon atom, formation energies depend on the growth conditions for these defects, i.e., N-rich (B-poor), B-rich (N-poor), or some intermediate conditions. The results for "extreme" conditions are shown in Fig. 2 and are in agreement with previous calculations, e.g., Refs. [24,25]. In addition to simple substitutional defects, in Fig. 2 we show the formation energies of carbon clusters with an unequal number of carbon atoms on boron or nitrogen sites: carbon trimers, pentamers, and triangular defects $\mathrm{C}_{\mathrm{N}}\left(\mathrm{C}_{\mathrm{B}}\right)_{3}$ and $C_{B}\left(C_{N}\right)_{3}$. In the formation energy plot, we depict only the most stable charge state for a given Fermi level. This yields a piecewise linear dependence of $\Delta H_{f}$ on $E_{F}$. The actual charge state can be read out from the slope of the line at a given $E_{F}$ [39].

Vertical dashed lines in Fig. 2 indicate the values of the calculated Fermi level. We choose $T=1600 \mathrm{~K}$ as a representative temperature (see Sec. V), but we find that $E_{F}$ depends quite weakly on $T$. The position of the Fermi level is shown for the growth with and without oxygen. Different situations will be discussed in Sec. IV B.

We find that, in general, formation energies increase with the number of carbon atoms in the cluster. Single substitutionals, as well as trimers and pentamers, can have three charge states: $-1,0$, and +1 . From the chemical perspective, one can expect that, for example, clusters with one additional $C_{N}$ unit are single acceptors. Therefore the existence of 0 and -1 charge states is natural. However, calculations show that a positive charge state is also stable in all cases. A similar conclusion holds for clusters with one more carbon atom on the boron site, where we also find three charge states. In the case of starlike defects, we also find higher charge states: +2 for $C_{N}\left(C_{B}\right)_{3}$ and -2 for $C_{B}\left(C_{N}\right)_{3}$. The latter defects were first considered theoretically in Refs. [46,47]. Our calculated formation energies are in good overall agreement with those calculations (apart from the existence of $q=-3$ charge states; see Ref. [48] for a discussion on this point). The differences with the results of Refs. [46,47] can be explained by the significantly larger supercells used in our work, as well as the electrostatic correction term $E_{\text {corr }}$ [Eq. (1)], which is especially important for higher charge states. Formation energies of trimers were first reported in Ref. [16], and our calculations are in good agreement with those results. We will label all defects, the formation energies of which are shown in Fig. 2, as "odd carbon clusters."

Even carbon clusters. Formation energies of carbon clusters with an equal number of carbon atoms on nitrogen and boron sites are the same for nitrogen-rich and nitrogen-poor conditions. We will label these clusters as "even carbon clusters." Formation energies for clusters $\mathrm{C}_{2}, \mathrm{C}_{4}, \mathrm{C}_{6}, \mathrm{C}_{8}$, and $\mathrm{C}_{10}$ are shown in Fig. 3. The result for the dimer has been previously presented in Ref. [25]. We find that in the case of all even carbon clusters, the neutral charge state is the most stable one throughout most of the band gap. The +1 and -1 charge states can be stabilized for Fermi levels close to the band edges. In our simulations, we find that even carbon clusters are always predominantly charge neutral for the actual Fermi levels.

Carbon clusters $\mathrm{C}_{n}$ with $n>3$ can have several nonidentical configurations. Formation energies of the most stable of these are shown in Figs. 2 and 3. Concentrations of only these most stable clusters are analyzed in Sec. IV B. For example, the hexamer $\mathrm{C}_{6}$ can exist either as an open chain or as a closed ring (an analog of the benzene molecule). The energies of these two configurations are different: The "benzene" 


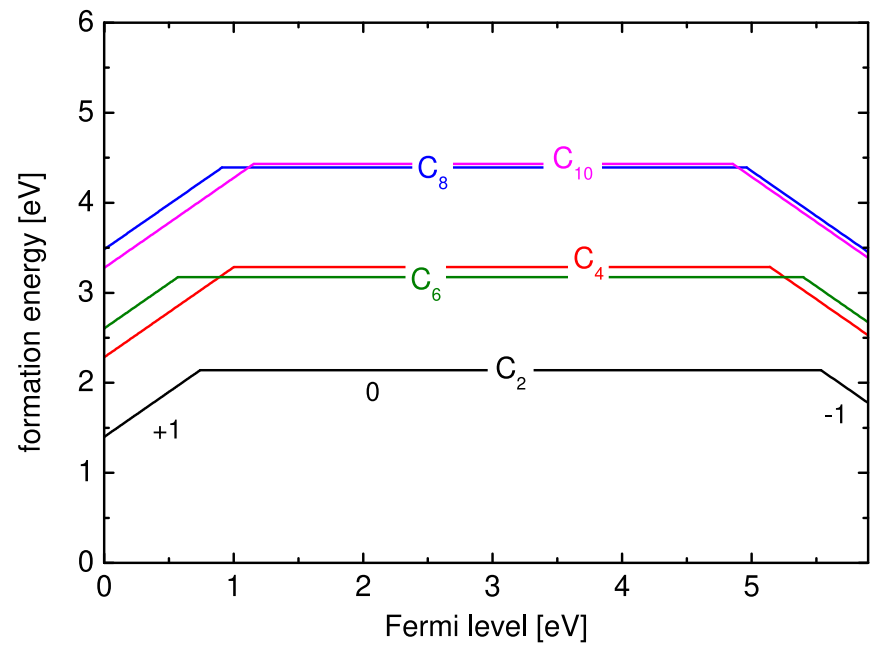

FIG. 3. Formation energy of even carbon clusters as a function of the Fermi level. Defects included: carbon dimer $\mathrm{C}_{2}$, tetramer $\mathrm{C}_{4}$, hexamer $\mathrm{C}_{6}$, octamer $\mathrm{C}_{8}$, and decamer $\mathrm{C}_{10}$.

configuration has the lowest energy, while the energy of an open chain is larger. A slightly different situation is encountered for $\mathrm{C}_{4}$. It can exist in either the cis or the trans configuration, both shown in Fig. 1. The energies of these two configurations are nearly equal, and both of them are included in our analysis. In general, we find that the formation energy of even carbon clusters in the neutral charge state is essentially determined by the number of $\mathrm{C}-\mathrm{N}$ and $\mathrm{C}-\mathrm{B}$ bonds (in the case of BCN alloys, an equivalent relationship was first pointed out in Ref. [49]). This simple chemical picture explains the nearly equal formation energies of both configurations of $\mathrm{C}_{4}$. It also explains the nearly equal formation energies of $\mathrm{C}_{4}$ and $\mathrm{C}_{6}$ (three pairs of $\mathrm{C}-\mathrm{N}$ and $\mathrm{C}-\mathrm{B}$ bonds), as well as the lowest-energy configurations of $\mathrm{C}_{8}$ and $\mathrm{C}_{10}$ (four pairs), evident from Fig. 3. The $\mathrm{C}_{8}$ complex with the lowest energy can be thought of as a carbon ring plus a carbon dimer attached to it. The lowest-energy configuration of the $\mathrm{C}_{10}$ complex is made of two carbon rings, an equivalent of the naphthalene molecule. When calculating the lattice multiplicity factor $g_{D}$, we consider only the lowest-energy configuration or, in the case of tetramers, the two configurations that have nearly equal energies. Other configurations with larger formation energies occur at smaller concentrations in comparison to the most stable configurations.

Oxygen-containing defects. Formation energies of the $\mathrm{C}_{\mathrm{N}} \mathrm{O}_{\mathrm{N}}$ defect are shown in Fig. 4 for both N-rich and N-poor conditions. Under $\mathrm{N}$-poor conditions, the formation energy of the neutral complex is very low, just $1.2 \mathrm{eV}$. Coulomb attraction between a positively charged oxygen and a negatively charged carbon explains such a low value. Indeed, by comparing the total energy of the separated $\mathrm{C}_{\mathrm{N}}-\mathrm{O}_{\mathrm{N}}$ pair and the $\mathrm{C}_{\mathrm{N}} \mathrm{O}_{\mathrm{N}}$ defect, we obtain a Coulomb stabilization energy of $1.5 \mathrm{eV}$. We can thus anticipate this defect to form in large concentrations whenever both oxygen and carbon are present under N-poor conditions. The formation energy is significantly larger under N-rich conditions. Formation energies of the considered oxygen defects not containing carbon are given in, e.g., Ref. [24].

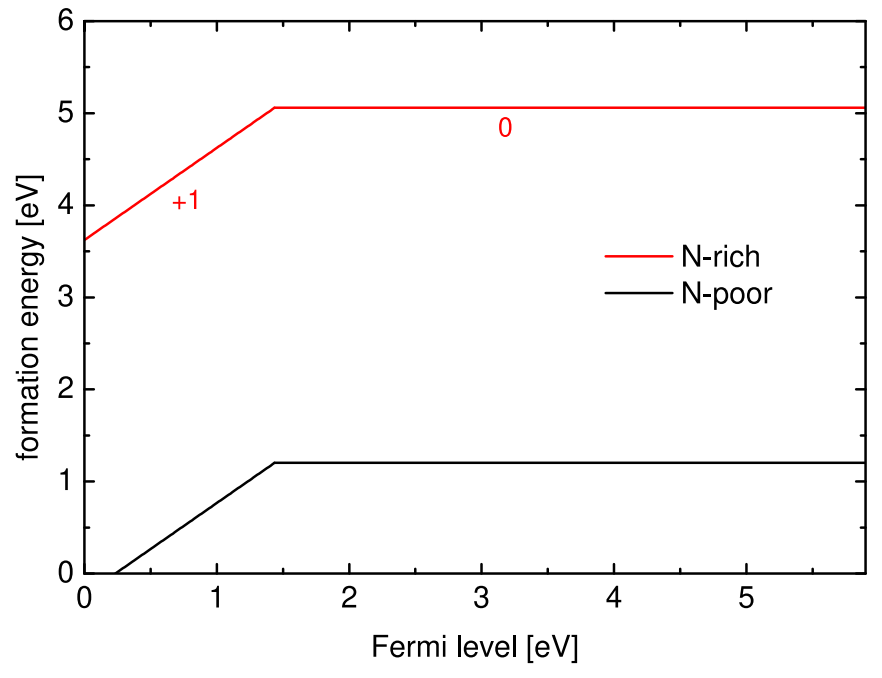

FIG. 4. Formation energy of the $\mathrm{C}_{\mathrm{N}} \mathrm{O}_{\mathrm{N}}$ defect as a function of the Fermi level for $\mathrm{N}$-rich and $\mathrm{N}$-poor conditions.

Other carbon defects. Under typical growth temperatures of hBN, carbon complexes with vacancies and antisites occur at concentrations smaller than $10^{14} \mathrm{~cm}^{-3}$, and their formation energies are given in the Supplemental Material [44]. The same is true for non-nearest-neighbor $C_{B}-C_{N}$ pairs, which are also analyzed in the Supplemental Material [44]. As discussed in Sec. IV B, the only exception is the $\mathrm{C}_{\mathrm{B}} V_{\mathrm{B}}$ pair. Its formation energy is also given in the Supplemental Material.

\section{B. Equilibrium densities of defects}

hBN with carbon only. Calculated densities of carbon defects in the absence of oxygen are shown in Figs. 5(a)-5(c) for temperatures up to $T=2200 \mathrm{~K}$. This range covers temperatures of the most popular growth techniques of hBN: 1550-1650 K for molecular beam epitaxy (MBE), about $1600 \mathrm{~K}$ for metalorganic vapor-phase epitaxy (MOVPE), and 1800-2200 K for bulk growth [13]. Figure 5(a) corresponds to $\mathrm{N}$-poor conditions, Fig. 5(c) corresponds to N-rich conditions, and Fig. 5(b) corresponds to the intermediate situation exactly at the midpoint between $\mathrm{N}$-rich and $\mathrm{N}$-poor. The densities shown in Fig. 5 reflect all charge states of defects. However, one charge state is often the most prevalent. We discuss the most prevalent charge state of defects below, and for illustration purposes, we choose the temperature $T=1600 \mathrm{~K}$.

Under N-poor conditions, the carbon on the nitrogen site $\mathrm{C}_{\mathrm{N}}$ is the most prevalent defect [Fig. 5(a)]. At $T=1600 \mathrm{~K}$, the Fermi level is about $1.9 \mathrm{eV}$ above the VBM [Fig. 2(a)]. Therefore, $C_{N}$ is mostly neutral. The concentration of carbon dimers $C_{2}$ is about 10 times smaller. Trimers $C_{2} C_{N}$ (predominantly in the neutral charge state) also occur in appreciable quantities. These defects are followed by positively charged $C_{B}$, neutral triangular defect $C_{B}\left(C_{N}\right)_{3}$, and neutral clusters $C_{4}$, $\mathrm{C}_{6}$, and $\mathrm{C}_{4} \mathrm{C}_{\mathrm{N}}$. Larger carbon clusters and all other carbonrelated defects occur in smaller densities.

The situation under N-rich conditions [Fig. 5(c)] approximately mirrors the situation under $\mathrm{N}$-poor conditions with the substitution $\mathrm{C}_{\mathrm{N}} \leftrightarrow \mathrm{C}_{\mathrm{B}}$. At $T=1600 \mathrm{~K}$, the Fermi level is about $4.7 \mathrm{eV}$ above the VBM, and the defect chemistry is 
without $\mathrm{O}$

(a) N-poor

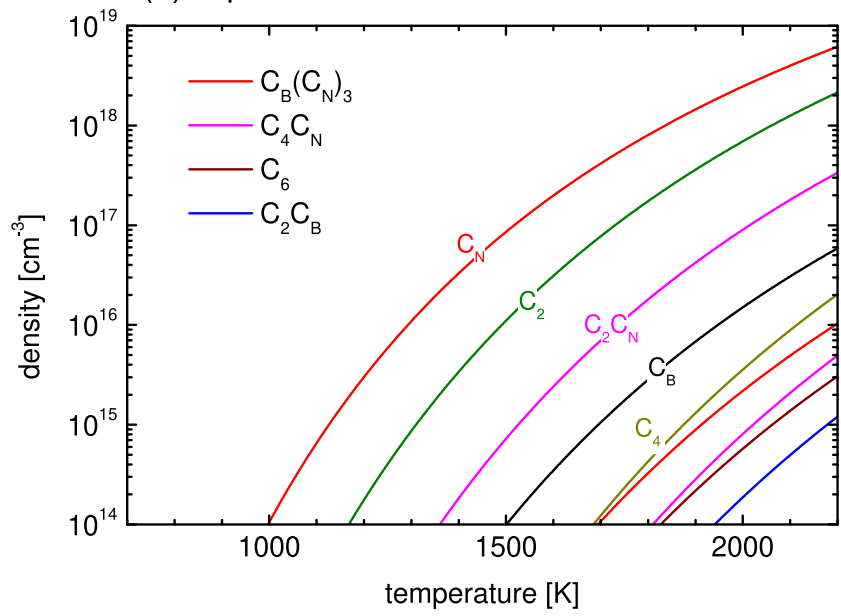

(b) intermediate

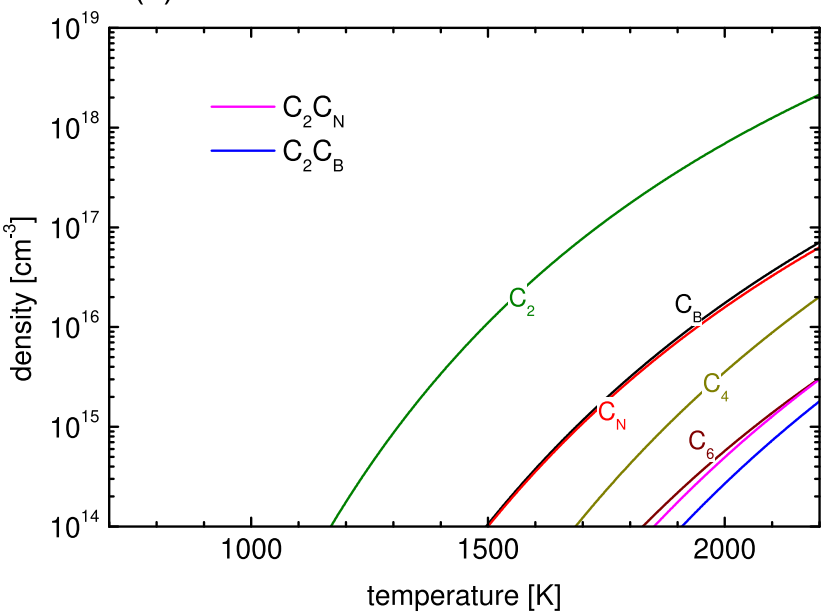

(c) N-rich

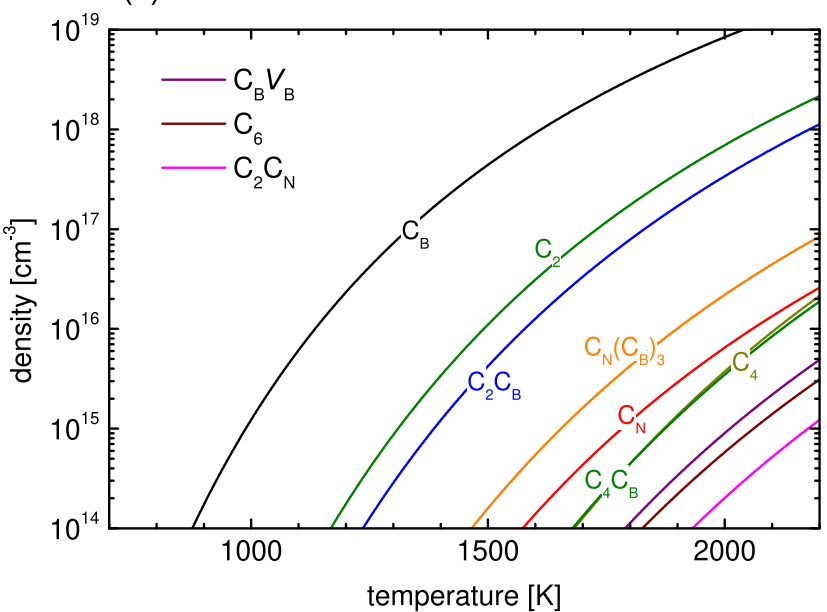

with $\mathrm{O}$

(d) N-poor

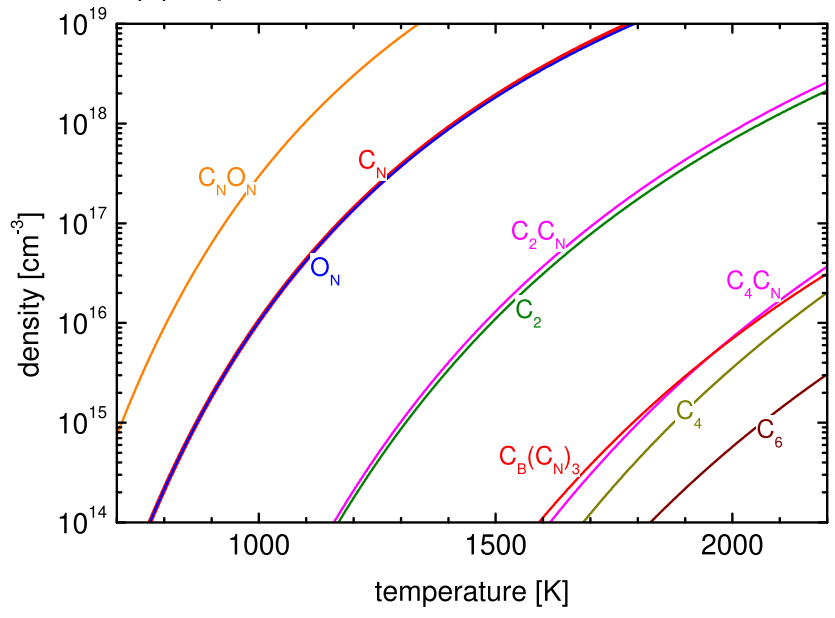

(e) intermediate

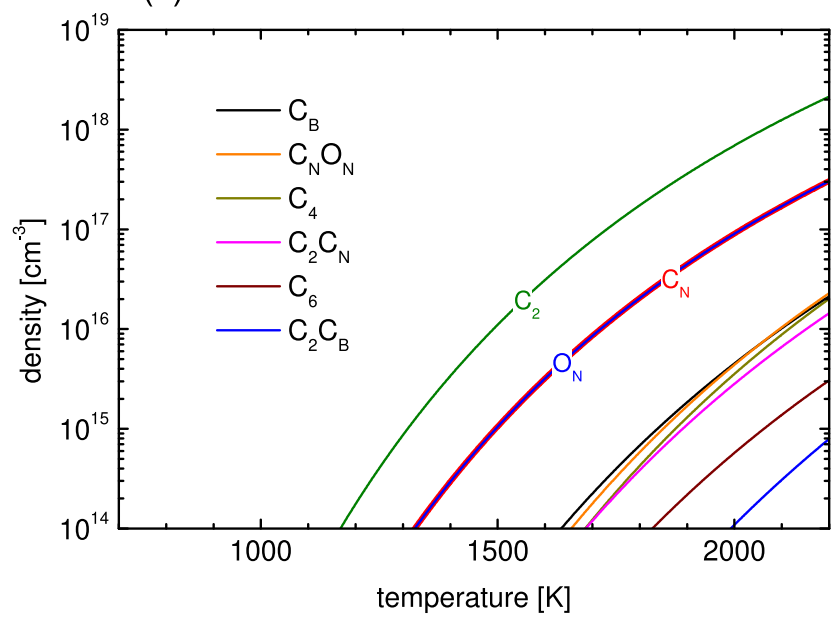

(f) N-rich

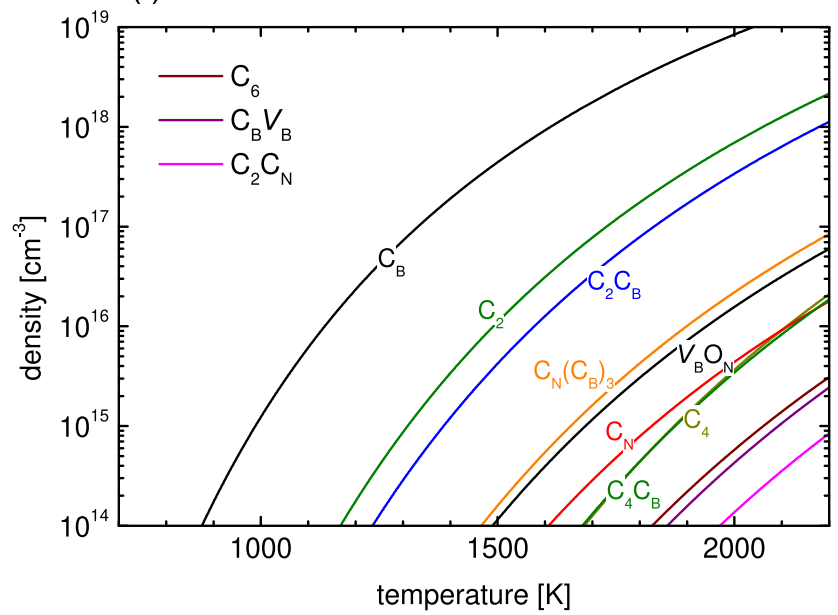

FIG. 5. Equilibrium concentration of defects as a function of temperature without [(a)-(c)] and with [(d)-(f)] the presence of oxygen during growth. (a) and (d) N-poor conditions, (b) and (e) intermediate conditions between N-poor and N-rich, and (c) and (f) N-rich conditions. Carbon-rich conditions are assumed. These conditions yield the maximum density of carbon defects. Only defects that attain concentrations $N_{D}>10^{14} \mathrm{~cm}^{-3}$ for growth temperatures below $T=2000 \mathrm{~K}$ are shown. 
dominated by neutral $\mathrm{C}_{\mathrm{B}}$. Neutral carbon dimers and neutral $\mathrm{C}_{2} \mathrm{C}_{\mathrm{B}}$ occur at smaller concentrations. These are followed by neutral starlike defects $C_{N}\left(C_{B}\right)_{3}$, negatively charged $C_{N}$, negatively charged $\mathrm{C}_{2} \mathrm{C}_{\mathrm{N}}$ trimers, and neutral clusters $\mathrm{C}_{4}, \mathrm{C}_{4} \mathrm{C}_{\mathrm{B}}$, and $\mathrm{C}_{6}$. A noticeable exception to the "mirror symmetry" is the $\mathrm{C}_{\mathrm{B}} V_{\mathrm{B}}$ defect. This defect occurs in the $q=-2$ charge state, and its concentration can exceed $10^{14} \mathrm{~cm}^{-3}$ (but only at growth temperatures $T>1800 \mathrm{~K}$ ).

Under intermediate conditions, the picture is rather different from the previous two scenarios [Fig. 5(b)]. At temperature $T=1600 \mathrm{~K}$, the Fermi level is $3.3 \mathrm{eV}$ above the VBM, i.e., close to the midgap. We find that carbon dimers $\mathrm{C}_{2}$ become the most dominant defects (their formation energies are independent of the chemical potentials of boron and nitrogen). The concentration of single substitutionals is about two orders of magnitude smaller (though still quite appreciable). Single substitutionals are followed by $\mathrm{C}_{4}$ and $\mathrm{C}_{6}$. As in the case of dimers, the formation energies of $\mathrm{C}_{4}$ and $\mathrm{C}_{6}$ clusters are independent of the boron and nitrogen chemical potentials (within the constraint of equilibrium growth, $\mu_{\mathrm{B}}+\mu_{\mathrm{N}}=$ $\left.\mu_{\mathrm{BN}}\right)$. Formation energies of these two defects are similar (Fig. 3), and larger densities of tetramers are explained by the difference in lattice multiplicity factors $g_{D}$ (6 for tetramers vs $1 / 2$ for hexamers). Under these intermediate conditions, carbon trimers appear in much smaller concentrations than under "extreme" N-poor or N-rich conditions. The concentration of triangular starlike defects falls significantly below our chosen threshold of $10^{14} \mathrm{~cm}^{-3}$.

$h B N$ with carbon and oxygen. The presence of oxygen changes the overall picture. The concentration of carbonand oxygen-containing point defects is shown in Figs. 5(d)5(f). The effect of oxygen is most pronounced under N-poor conditions [Fig. 5(d)]. It manifests itself (i) via the large concentration of oxygen-containing defects and (ii) via a change in concentrations of carbon defects not containing oxygen. Due to the very low formation energy of the $\mathrm{C}_{\mathrm{N}} \mathrm{O}_{\mathrm{N}}$ defect under $\mathrm{N}$-poor conditions (Fig. 4), $\mathrm{C}_{\mathrm{N}} \mathrm{O}_{\mathrm{N}}$ occurs in concentrations exceeding $10^{19} \mathrm{~cm}^{-3}$ for growth temperatures $T>1300 \mathrm{~K}$. This defect occurs primarily in the neutral charge state and does not influence the position of the Fermi level. However, the Fermi level is affected by the incorporation of oxygen donors $\mathrm{O}_{\mathrm{N}}$ that also occur in substantial concentrations. In comparison to the system without oxygen, the Fermi level is pushed up by more than $1 \mathrm{eV}$ [Fig. 2(a)]; $E_{F}$ is now determined by the equilibrium between positively charged donors $\mathrm{O}_{\mathrm{N}}$ and negatively charged acceptors $\mathrm{C}_{\mathrm{N}}$. As a result, the concentration of $\mathrm{C}_{\mathrm{N}}$ also increases (due to an increase in negatively charged species; the concentration of neutral $C_{N}$ is the same as in the case without oxygen). The raising of the Fermi level does not affect the concentration of even carbon clusters. However, higher Fermi levels increase the concentration of negatively charged odd carbon clusters with surplus $\mathrm{C}_{\mathrm{N}}$ units and decrease the concentration of positively charged carbon clusters with surplus $\mathrm{C}_{\mathrm{B}}$ units. Most importantly, we find that the concentration of carbon trimers $\mathrm{C}_{2} \mathrm{C}_{\mathrm{N}}$ becomes quite appreciable, very close to that of dimers. Similarly, concentrations of starlike defects $\mathrm{C}_{\mathrm{B}}\left(\mathrm{C}_{\mathrm{N}}\right)_{3}$ and pentamers $\mathrm{C}_{4} \mathrm{C}_{\mathrm{N}}$ are boosted.

In contrast to $\mathrm{N}$-poor conditions, oxygen does not significantly affect the formation of carbon defects under N-rich conditions [Fig. 5(f)]. The densities of carbon defects remain similar to the case without oxygen [Fig. 5(c)].

Under intermediate conditions between N-rich and N-poor [Fig. 5(e)], the situation resembles that with no oxygen. The presence of oxygen raises the Fermi level by about $0.3 \mathrm{eV}$, and $E_{F}$ nearly reaches the $\mathrm{C}_{\mathrm{B}}$ defect's $(+/ 0)$ charge-state transition level. The consequence of this is a smaller concentration of $C_{B}$ defects, and charge neutrality is established by the equilibrium between positively charged $\mathrm{O}_{\mathrm{N}}$ donors and negatively charged carbon acceptors $\mathrm{C}_{\mathrm{N}}$. A modest increase in the Fermi level slightly increases the concentration of carbon defects with surplus $\mathrm{C}_{\mathrm{N}}$ units and decreases the concentrations of those with surplus $C_{B}$ units. Regarding carbon defects, one could conclude that the influence of oxygen is not particularly strong in this regime [cf. Figs. 5(b) and 5(e)].

\section{DEFECT THERMODYNAMICS AND SINGLE-PHOTON EMISSION IN CARBON-DOPED hBN}

In this section, we discuss the findings of Ref. [13] in light of the results presented in Sec. IV B. We will mostly focus on MOVPE samples that were grown at $~ 1600 \mathrm{~K}$ [13]. For these samples, carbon was introduced via the triethylborane (TEB) precursor, the flow rate of which could be varied. The resulting epitaxial layers were about $h=40 \mathrm{~nm}$ thick. Altering the flow rate of the carbon-carrying precursor effectively changes the chemical potential of carbon. The highest chemical potential that can be achieved is determined by the onset of formation of bulk inclusions of graphite (see Sec. VI). This is the carbon-rich limit, whereby the chemical potential of carbon is determined by the energy of one $\mathrm{C}$ atom in graphite. It was exactly this limit that was assumed in the calculation of formation energies and concentrations in Sec. IV. This limit determines the maximum possible concentration of carbon-related point defects. When the flow of the precursor is lowered, the carbon chemical potential decreases, which increases the formation energy of carbon-related defects, leading to their smaller concentrations.

In Ref. [13], the authors could detect isolated carbonrelated SPEs, emitting in the energy range $1.6-2.1 \mathrm{eV}$, for the lowest flow rate of the precursor TEB $(10 \mu \mathrm{mol} / \mathrm{min})$. However, single emitters could no longer be identified for higher flow rates of the TEB precursor $(\geqslant 20 \mu \mathrm{mol} / \mathrm{min})$, as the density of emitters became too large. The emitters can no longer be identified as single ones when their lateral density becomes larger than one emitter per diffraction spot of the laser. The diameter of the laser spot can be assumed to be equal to the diameter of the Airy disk, $d=1.22 \lambda / \mathrm{NA}$, where $\lambda$ is the wavelength of the laser and NA is the numerical aperture. Using the parameters of Ref. [13], $\lambda=532 \mathrm{~nm}$ and $\mathrm{NA}=0.95$, we obtain $d \approx 680 \mathrm{~nm}$. The total volume per one emitter is then smaller than $\pi d^{2} h / 4$, yielding 3D concentrations larger than $0.7 \times 10^{14} \mathrm{~cm}^{-3}$

This means that the emitters observed in Ref. [13] are defects that can occur in concentrations larger than about $N_{D}=$ $10^{14} \mathrm{~cm}^{-3}$. This was exactly the number cited in Sec. IV and chosen as our criterion. However, one must keep in mind that it is not entirely clear whether the maximum flow rate of the carbon precursor used in Ref. [13] indeed corresponds to the carbon-rich limit. If it does not, then the carbon-rich 
limit would yield even higher defect concentrations than the ones obtained in Ref. [13]. This makes our chosen criterion $N_{D}=10^{14} \mathrm{~cm}^{-3}$ a good measure regarding possible defect centers to explain the results of Ref. [13].

\section{A. hBN with carbon only}

Let us now analyze the results of our calculations shown in Fig. 5 and compare them with the results of Ref. [13]. We will first consider the situation without oxygen present.

$\mathrm{N}$-poor conditions. In the case of $\mathrm{N}$-poor conditions [Fig. 5(a)], we see that only a few carbon-related defects can occur at concentrations larger than $10^{14} \mathrm{~cm}^{-3}$ for growth temperatures $T=1600 \mathrm{~K}$. These defects are: $\mathrm{C}_{\mathrm{N}}$, the dimer $\mathrm{C}_{2}$, the trimer $\mathrm{C}_{2} \mathrm{C}_{\mathrm{N}}$ (all charge neutral), and positively charged $\mathrm{C}_{\mathrm{B}}$. The neutral triangular defect $\mathrm{C}_{\mathrm{B}}\left(\mathrm{C}_{\mathrm{N}}\right)_{3}$ also approaches threshold concentrations. Historically, $\mathrm{C}_{\mathrm{N}}$ was associated [23] with the ubiquitous 4.1-eV luminescence line in hBN. However, density functional theory calculations showed that this attribution was not correct [24]. In recent theoretical work, Auburger and Gali calculated the ZPL energy of the neutral $\mathrm{C}_{\mathrm{N}}$ to be about $2.5 \mathrm{eV}$ [17], not far from the experimentally measured values of visible SPEs. The optical transition occurs from a localized defect level to a perturbed bulk state, stabilized by an electron-hole interaction energy of $\approx 0.8 \mathrm{eV}$. This defect, however, possesses a $D_{3 h}$ point group symmetry. As a result, there should not be a fixed emission dipole in the $\mathrm{hBN}$ plane for dipole-allowed optical transitions. Instead, the emission could only have random polarization in the $\mathrm{hBN}$ plane. This is in contradiction to experiments that show a fixed dipole [11]. However, local electric or strain fields can lower the symmetry and lock the direction of the transition dipole moment with respect to the underlying lattice.

There is firm theoretical evidence that the carbon dimer defect emits in the UV region, likely being the cause of the 4.1-eV emission [25,50,51]. Our calculations using the socalled delta-self-consistent-field $(\triangle \mathrm{SCF})$ approach [52] yield the value of $4.12 \mathrm{eV}$, in excellent agreement with the experimental result. Our thermodynamic modeling indicates that the concentration of dimers should exceed $10^{16} \mathrm{~cm}^{-3}$ in MOVPE samples, meaning that dimers are indeed ubiquitous. Regarding the optical signature of the neutral trimer $\mathrm{C}_{2} \mathrm{C}_{\mathrm{N}}$, it was recently proposed that its theoretical luminescence lineshape strongly resembles that of some 1.6-2.1-eV SPEs [15]. Calculations of Refs. [15,17] yield the value of $1.62 \mathrm{eV}$ for the ZPL energy, indeed close to the experimental range. Our thermodynamical calculations confirm that this defect is a contender to explain single-photon emission in the IR-visible energy range.

Positively charged $C_{B}$ defects that occur in smaller concentrations than neutral trimers $\mathrm{C}_{2} \mathrm{C}_{\mathrm{N}}$ do not have filled defect states and therefore should not have an internal defect transition.

Finally, to the best of our knowledge, optical properties of the triangular defect $\mathrm{C}_{\mathrm{B}}\left(\mathrm{C}_{\mathrm{N}}\right)_{3}$ have not been investigated so far. Whenever this defect occurs in significant concentrations ("extreme" N-poor conditions), it is the neutral charge state that is the most prevalent. The ground state of the defect has spin $S=1$. A preliminary analysis of the electronic structure of this defect reveals that the excited state is of multideterminant nature. As a result, it is not straightforward to obtain the
ZPL energy using $\triangle \mathrm{SCF}$ calculations. However, the nature of the excited state is expected to be similar to that of the $\mathrm{C}_{\mathrm{N}}$ defect, analyzed in Ref. [17]. Thus we can make an estimate of the ZPL energy of this defect by determining the energy needed to excite a hole to a valence band and taking into consideration the electron-hole interaction energy of $\approx 0.8 \mathrm{eV}$. In this way we obtain a ZPL energy of $\approx 2.2 \mathrm{eV}$, very close to the energy of visible emitters in hBN. Due to its triplet ground state, the $C_{B}\left(C_{N}\right)_{3}$ defect is potentially an interesting center in $\mathrm{hBN}$, worth further investigation. From the thermodynamical standpoint, our results show that this defect can occur in sufficient concentrations under N-poor conditions. Based both on the thermodynamical modeling and an estimate of the ZPL energy, we cannot exclude this defect as contributing to single-photon emission in the 1.6-2.1-eV energy range. However, like the $\mathrm{C}_{\mathrm{N}}$ defect, the $\mathrm{C}_{\mathrm{B}}\left(\mathrm{C}_{\mathrm{N}}\right)_{3}$ center possesses $D_{3 h}$ symmetry. Thus, optical polarization of emission could have a random orientation in the $\mathrm{hBN}$ plane, but should not have a fixed in-plane transition dipole moment in the absence of other perturbations.

$N$-rich conditions. The situation for N-rich conditions [Fig. 5(c)] is in a way symmetrical to that of N-poor conditions under substitution $\mathrm{C}_{\mathrm{N}} \leftrightarrow \mathrm{C}_{\mathrm{B}}$. The most dominant defect is neutral $\mathrm{C}_{\mathrm{B}}$. Auburger and Gali obtained a ZPL energy of about $1.7 \mathrm{eV}$ for this defect, falling in the experimental range for visible-IR SPEs [17]. Luminescence corresponds to a transition of the electron from a perturbed conduction band state, stabilized by electron-hole interaction, to a localized defect state. In terms of abundance, this defect is followed by the carbon dimer and the trimer $\mathrm{C}_{2} \mathrm{C}_{\mathrm{B}}$. The calculated optical signature of this trimer is similar to that of $C_{2} C_{N}$ [15]. The calculations of Ref. [15] yield a ZPL value of $1.65 \mathrm{eV}$, while a smaller value of $1.36 \mathrm{eV}$ was obtained in Ref. [17]. These results hint that the $\mathrm{C}_{2} \mathrm{C}_{\mathrm{B}}$ defect is a less likely contender to explain emission in the 1.6-2.1-eV energy range. However, keeping in mind possible errors in the evaluation of optical transition energies, based on thermodynamics alone, we expect that the $\mathrm{C}_{2} \mathrm{C}_{\mathrm{B}}$ trimer can also be a potential candidate to explain single-photon emission in the red and near infrared.

The triangular defect $\mathrm{C}_{\mathrm{N}}\left(\mathrm{C}_{\mathrm{B}}\right)_{3}$ occurs in rather large concentrations $\left(\sim 10^{15} \mathrm{~cm}^{-3}\right)$ at growth temperatures of $1600 \mathrm{~K}$. As in the case of the $C_{B}\left(C_{N}\right)_{3}$ defect, we find that the neutral charge state is the most relevant one. The ground state of $\mathrm{C}_{\mathrm{N}}\left(\mathrm{C}_{\mathrm{B}}\right)_{3}$ is spin triplet $(S=1)$. We can estimate the ZPL energy of this defect similarly to the $\mathrm{C}_{\mathrm{B}}\left(\mathrm{C}_{\mathrm{N}}\right)_{3}$ defect. We obtain an energy of $\approx 2.0 \mathrm{eV}$. Due to its triplet ground state, the $\mathrm{C}_{\mathrm{N}}\left(\mathrm{C}_{\mathrm{B}}\right)_{3}$ triangular defect is also a potentially interesting system for quantum applications. Based on thermodynamical modeling and the estimate of the ZPL energy, we suggest this defect as a potential candidate to explain single-photon emission in the $1.6-2.1-\mathrm{eV}$ energy range. These preliminary estimates call for a more rigorous investigation of the electronic structure and optical transitions of both triangular starlike defects, which should be the focus of future work.

Intermediate conditions. At conditions intermediate between $\mathrm{N}$-rich and $\mathrm{N}$-poor, the concentration of trimers and triangular defects falls significantly below $10^{14} \mathrm{~cm}^{-3}$, and carbon chemistry is dominated by carbon dimers. Apart from 
dimers and single substitutional defects, no other carbonrelated defects form in large enough concentrations.

Bulk samples. Bulk samples are grown at temperatures $T=1800-2200 \mathrm{~K}$ [5]. Figure 5 indicates that under these conditions the formation of larger carbon clusters, in particular tetramers $\mathrm{C}_{4}$ and hexamers $\mathrm{C}_{6}$, becomes more pronounced and exceeds $10^{14} \mathrm{~cm}^{-3}$. In MOVPE samples grown at lower temperatures the concentration of these larger clusters should be about $10^{13} \mathrm{~cm}^{-3}$. In addition to these defects, carbon pentamers $\mathrm{C}_{4} \mathrm{C}_{\mathrm{N}}$ are quite common under $\mathrm{N}$-poor conditions, while $\mathrm{C}_{4} \mathrm{C}_{\mathrm{B}}$ show up under $\mathrm{N}$-rich conditions. The existence of carbon hexamers, along with dimers, was indeed confirmed in Ref. [7], where authors used annular dark-field electron microscopy to image hBN monolayers exfoliated from bulk samples.

Optical emission energies of these defects are not known. In the case of the $\mathrm{C}_{4}$ defect, we determine the ZPL energy using the aforementioned $\triangle \mathrm{SCF}$ calculations. Since the excited state is a spin singlet, we have to apply a singlet correction as has been discussed in, e.g., Ref. [25]. We obtain a ZPL energy of $3.10 \mathrm{eV}$ for the trans isomer and $3.45 \mathrm{eV}$ for the cis isomer. Unfortunately, $\triangle \mathrm{SCF}$ calculations cannot be straightforwardly applied for the $\mathrm{C}_{6}$ defect due to its more complicated electronic structure. A simple estimate can be made based on our calculated Kohn-Sham energy levels of the $\mathrm{C}_{6}$ defect and the comparison with the dimer defect, for which the ZPL energy can be calculated. Using this approximation, we can estimate that the ZPL energy of the carbon hexamer should be about $4.0 \mathrm{eV}$. Similarly, we estimate that the ZPL energy of the $C_{4} C_{N}$ pentamer should be about $0.15 \mathrm{eV}$ smaller than the corresponding energy of the $\mathrm{C}_{2} \mathrm{C}_{\mathrm{N}}$ trimer, and that of the $\mathrm{C}_{4} \mathrm{C}_{\mathrm{B}}$ pentamer should be about $0.15 \mathrm{eV}$ smaller than the ZPL energy of the $\mathrm{C}_{2} \mathrm{C}_{\mathrm{B}}$ trimer.

In addition to these larger carbon clusters, a doubly negatively charged $C_{B} V_{B}$ complex can appear in sufficient concentrations in bulk samples grown under N-rich conditions. In their paper, Korona and Chojecki [53] studied optical transitions of the neutral center and suggested ZPL energies of about $1 \mathrm{eV}$. However, we find that the doubly negatively charged center, which is the relevant charge state, does not have an internal optical transition, as all of the defect states in the band gap are filled. Therefore this defect is an unlikely candidate to contribute to single-photon emission in the energy range $1.6-2.1 \mathrm{eV}$.

\section{B. hBN with carbon and oxygen}

As discussed in Sec. IV B, the presence of oxygen most strongly affects carbon defect concentrations under N-poor conditions. The largest difference is the occurrence of the $\mathrm{C}_{\mathrm{N}} \mathrm{O}_{\mathrm{N}}$ defect, which can form in amounts exceeding any other carbon and oxygen defects. It was proposed [26] that this defect causes the aforementioned 4.1-eV luminescence line, rivaling the carbon dimer hypothesis [25]. The concentrations of these defects, however, are drastically different in different hBN samples. $\mathrm{C}_{\mathrm{N}} \mathrm{O}_{\mathrm{N}}$ is clearly dominant under "extreme" $\mathrm{N}$-poor conditions, but the density of this defect falls quickly with the increase in the nitrogen chemical potential. At variance, the concentration of carbon dimers depends only on the chemical potential of carbon and is the same under $\mathrm{N}$-poor and
$\mathrm{N}$-rich conditions. This distinction is an important handle that could potentially help differentiate between the two defects.

The presence of oxygen does not affect our conclusions regarding SPEs in the energy range 1.6-2.1 eV. As already discussed, oxygen causes increased densities of $\mathrm{C}_{\mathrm{N}}$ defects and odd carbon clusters with surplus $\mathrm{C}_{\mathrm{N}}$ units (under N-poor conditions). However, no other defects apart from the ones analyzed above occur at concentrations higher than $10^{14} \mathrm{~cm}^{-3}$.

\section{VALIDITY OF THE FORMATION ENERGY FORMALISM}

Our analysis above relied on the use of the formation energy formalism, outlined in Sec. II. The key aspect of this formalism is that an external carbon source sets the carbon chemical potential $\mu_{\mathrm{C}}$. Setting $\mu_{\mathrm{C}}$ to the energy of carbon in graphite defines the maximum value of the carbon chemical potential. These are carbon-rich conditions that yield the maximum possible concentration of carbon-containing defects. Assuming a fixed chemical potential seems a natural choice when modeling epitaxial growth, e.g., MOVPE or MBE.

At variance, bulk growth occurs in closed crucibles with a fixed amount of different chemical species, in our case, boron, nitrogen, and carbon (and oxygen or other impurities, depending on conditions). In this case, the formation energy formalism to compute concentrations of point defects can still be applied. However, chemical potentials, including that of carbon, are not determined by external sources, but are governed by the total amount of different chemical species in the growth chamber.

Let us consider $\mathrm{hBN}$ with a certain density of carbon $N_{\mathrm{C}}$ at a temperature $T$. When $N_{\mathrm{C}}$ is sufficiently low, all the carbon (under thermodynamic equilibrium conditions) is in the form of point defects. The maximum amount of carbon that can exist in the form of point defects is given by the expression

$$
N_{\mathrm{C}, \max }(T)=\sum_{D} n_{\mathrm{C}} N_{D, \max }(T) .
$$

Here, the sum runs over all carbon-containing defects. $N_{D, \max }$ is the equilibrium density of defect $D$ in all charge states calculated for carbon-rich conditions, $N_{D, \max }(T)=$ $\sum_{q} N_{D, q, \max }(T)$ (the same as given in Fig. 5; here, we added the subscript "max" to emphasize that these are carbon-rich conditions). $N_{\mathrm{C}, \max }(T)$ can thus be thought of as the solubility limit of carbon in $\mathrm{hBN}$ when carbon is in the form of point defects.

When the actual amount of carbon is below the solubility limit, the procedure to determine the value of the carbon chemical potential $\mu_{\mathrm{C}}$ is as follows. Formation energies of point defects are determined by Eq. (1). The concentrations of defects are given by Eq. (2). The carbon chemical potential is determined by imposing the condition $\sum_{D} n_{\mathrm{C}} N_{D}(T)=N_{\mathrm{C}}$. The three equations have to be solved self-consistently (in addition to the equation to determine the electron chemical potential), which in the end yields both the actual value of $\mu_{\mathrm{C}}$ and the concentration of different carbon species. The procedure has been previously applied to other systems [54] and is illustrated quantitatively in the Supplemental Material [44]. When the amount of carbon exceeds $N_{\mathrm{C}, \max }(T)$, the carbon chemical potential corresponds to the carbon-rich limit, and 
(a) without $\mathrm{O}$

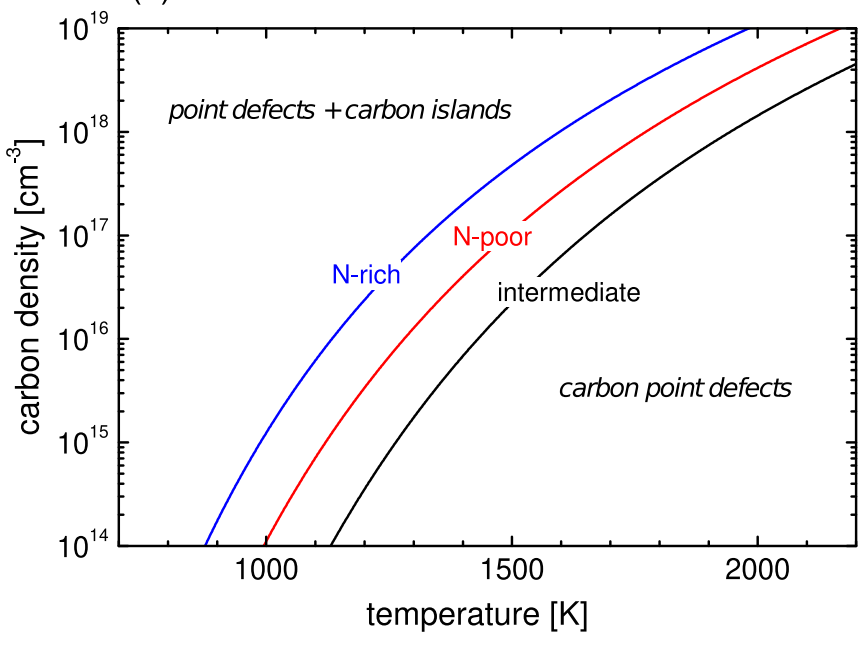

(b) with $\mathrm{O}$

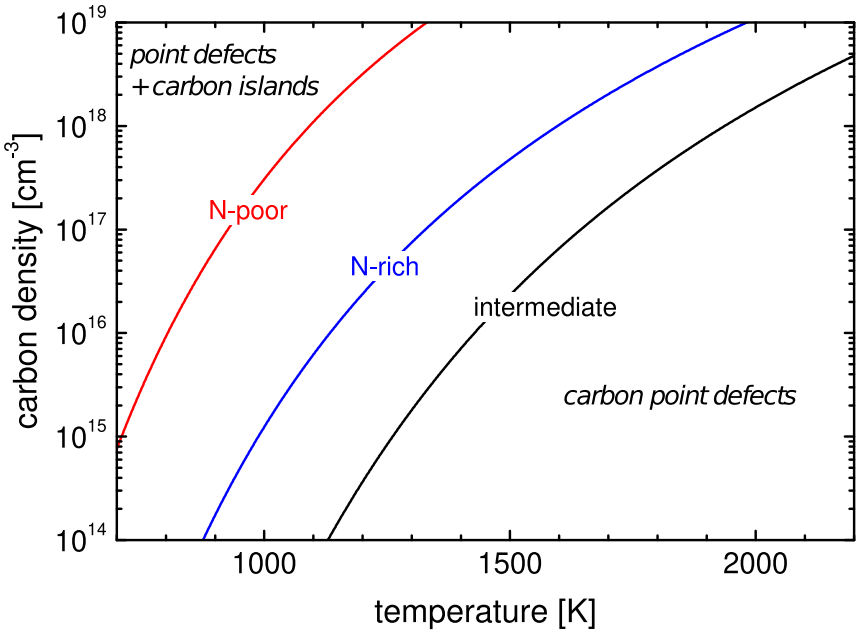

FIG. 6. Solubility limit of carbon in $\mathrm{hBN}$ as a function of temperature for $\mathrm{N}$-poor conditions, intermediate conditions between $\mathrm{N}$-poor and N-rich, and N-rich conditions. (a) Corresponds to growth without oxygen; (b) corresponds to growth with oxygen. The solubility limit is equal to the maximum density of carbon atoms that can be incorporated into hBN in the form of point defects. For carbon densities above the solubility limit, carbon starts to condense into graphite islands.

the concentrations of point defects are the ones that are shown in Fig. 5. The surplus carbon condenses into graphite islands.

The calculated solubility limits of carbon in $\mathrm{hBN}$ for different conditions, calculated using Eq. (3), are shown in Fig. 6. Figure 6(a) depicts the solubility limit without the presence of oxygen. The solubility limit is slightly larger for "extreme" $\mathrm{N}$-rich and $\mathrm{N}$-poor conditions and smaller for intermediate conditions. The situation is very different when oxygen is present, as shown in Fig. 6(b). The solubility limit is not much affected by oxygen under $\mathrm{N}$-rich and intermediate conditions but, in comparison to oxygen-free growth, is significantly increased for $\mathrm{N}$-poor conditions. This is mostly because of a very large concentration of $\mathrm{C}_{\mathrm{N}} \mathrm{O}_{\mathrm{N}}$ defects.

Figure 6 can also be viewed as the phase diagram of carbon in $\mathrm{hBN}$ in the dilute limit. In this point of view, the solubility limit is the binodal curve, above which the condensation of carbon ensues.

\section{CONCLUSIONS}

In this paper, we presented the thermodynamic analysis of carbon defects in hexagonal boron nitride. Our particular focus was the relevance of these results to single-photon emission in $\mathrm{hBN}$, where the involvement of carbon defects was reported [13].

Regarding carbon defects themselves, the conclusions of our work are the following:

(i) Under most growth conditions, when no oxygen is present, single substitutional defects $C_{N}$ and $C_{B}$, as well as carbon dimers $\mathrm{C}_{2}$, are carbon defects that occur in the largest concentrations. Theoretical calculations suggest that the ZPL energy of the $\mathrm{C}_{2}$ defect is about $4.1 \mathrm{eV}$, while ZPL energies of $C_{B}$ and $C_{N}$ defects are around 1.7 and $2.5 \mathrm{eV}$, respectively [17]. From the perspective of thermodynamics, the latter two defects are potential candidates to explain singlephoton emission in the energy region $1.6-2.1 \mathrm{eV}$, observed in Ref. [13].

(ii) Carbon trimers $\mathrm{C}_{2} \mathrm{C}_{\mathrm{B}}$ and $\mathrm{C}_{2} \mathrm{C}_{\mathrm{N}}$ occur in smaller concentrations than single substitutionals and dimers. However, their densities can be larger than $10^{14} \mathrm{~cm}^{-3}$ under certain growth conditions. In particular, $\mathrm{C}_{2} \mathrm{C}_{\mathrm{B}}$ defects should be abundant under $\mathrm{N}$-rich conditions, while $\mathrm{C}_{2} \mathrm{C}_{\mathrm{N}}$ should be commonplace under $\mathrm{N}$-poor conditions. Recent theoretical calculations showed that the ZPL energies of these defects are smaller than $1.7 \mathrm{eV}[15,17]$. Based on thermodynamical analysis and considering possible errors in evaluating optical transition energies, we infer that trimers can be deemed likely candidates to explain single-photon emission in the red and near-infrared spectral regions. Notwithstanding, the concentration of trimers is expected to be significantly smaller under intermediate conditions between N-rich and N-poor. Changing the chemical potentials of boron and nitrogen is thus a handle that can strongly affect the incorporation of these defects.

(iii) Triangular starlike defects $\mathrm{C}_{\mathrm{B}}\left(\mathrm{C}_{\mathrm{N}}\right)_{3}$ and $\mathrm{C}_{\mathrm{N}}\left(\mathrm{C}_{\mathrm{B}}\right)_{3}$ can also occur in concentrations approaching $10^{14} \mathrm{~cm}^{-3}$, but only under appropriate "purely" N-rich or N-poor conditions. For small deviations from these limit conditions, the formation energies of these defects increase substantially, and their concentration plummets. Neutral charge states of these defects are the relevant ones. $\mathrm{C}_{\mathrm{B}}\left(\mathrm{C}_{\mathrm{N}}\right)_{3}$ is expected to have a $\mathrm{ZPL}$ energy of $\approx 2.2 \mathrm{eV}$, while that of the $\mathrm{C}_{\mathrm{N}}\left(\mathrm{C}_{\mathrm{B}}\right)_{3}$ defect should be $\approx 2.0 \mathrm{eV}$. In contrast to other carbon defects discussed in this paper, both starlike defects have a triplet ground state $(S=1)$ [47], which makes them potentially useful for applications such as quantum sensing. Starlike defects are therefore interesting systems that require further studies.

(iv) Carbon tetramers $\mathrm{C}_{4}$ and hexamers $\mathrm{C}_{6}$ are also rather prominent defects. Their concentration should be around $10^{13} \mathrm{~cm}^{-3}$ in MBE and MOVPE samples grown under carbon-rich conditions but can surpass $10^{14} \mathrm{~cm}^{-3}$ in bulk hBN grown at about $2000 \mathrm{~K}$. Carbon hexamers are expected to have ZPL energies of $\approx 4.0 \mathrm{eV}$, i.e., very similar to carbon dimers. Computed ZPL energies of carbon tetramers are 3.10 and $3.45 \mathrm{eV}$. The fact that such large atomic clusters have sufficiently low formation energies and can form in significant 
TABLE I. Carbon-related defects with maximum equilibrium densities. Densities (in $\mathrm{cm}^{-3}$ ) correspond to growth temperatures $T=$ $1600 \mathrm{~K}$ and no oxygen present during growth. ZPL energies $E_{\mathrm{ZPL}}$ of internal defect transitions are in eV. Values of $E_{\mathrm{ZPL}}$ are either taken from published theoretical calculations, calculated in this paper, or estimated as described in the text (the latter case is distinguished by a symbol “ $\approx$ " before a number). All energies are approximated to within $0.1 \mathrm{eV}$. Defects are charge neutral unless explicitly stated.

\begin{tabular}{|c|c|c|c|c|c|c|c|c|c|}
\hline \multicolumn{5}{|c|}{ N-poor conditions } & \multicolumn{5}{|c|}{ N-rich conditions } \\
\hline Defect & Max. density & Symmetry & Spin & $E_{\mathrm{ZPL}}$ & Defect & Max. density & Symmetry & Spin & $E_{\mathrm{ZPL}}$ \\
\hline $\mathrm{C}_{\mathrm{N}}$ & $2 \times 10^{17}$ & $D_{3 h}$ & $1 / 2$ & $2.5[17]$ & $\mathrm{C}_{\mathrm{B}}$ & $1 \times 10^{18}$ & $D_{3 h}$ & $1 / 2$ & $1.7[17]$ \\
\hline $\mathrm{C}_{2}$ & $3 \times 10^{16}$ & $C_{2 v}$ & 0 & 4.1 & $\mathrm{C}_{2}$ & $3 \times 10^{16}$ & $C_{2 v}$ & 0 & 4.1 \\
\hline $\mathrm{C}_{2} \mathrm{C}_{\mathrm{N}}$ & $2 \times 10^{15}$ & $C_{2 v}$ & $1 / 2$ & $1.6[15,17]$ & $\mathrm{C}_{2} \mathrm{C}_{\mathrm{B}}$ & $1 \times 10^{16}$ & $C_{2 v}$ & $1 / 2$ & $1.7[15], 1.4$ [17] \\
\hline $\mathrm{C}_{\mathrm{B}}^{+}$ & $3 \times 10^{14}$ & $D_{3 h}$ & 0 & & $\mathrm{C}_{\mathrm{N}}\left(\mathrm{C}_{\mathrm{B}}\right)_{3}$ & $5 \times 10^{14}$ & $D_{3 h}$ & 1 & $\approx 2.0$ \\
\hline $\mathrm{C}_{\mathrm{B}}\left(\mathrm{C}_{\mathrm{N}}\right)_{3}$ & $3 \times 10^{13}$ & $D_{3 h}$ & 1 & $\approx 2.2$ & $\mathrm{C}_{\mathrm{N}}^{-}$ & $1 \times 10^{14}$ & $D_{3 h}$ & 0 & \\
\hline $\mathrm{C}_{4}$ & $3 \times 10^{13}$ & $C_{s}$ & 0 & 3.1 (trans), 3.5 (cis) & $\mathrm{C}_{4}$ & $3 \times 10^{13}$ & $C_{s}$ & 0 & 3.1 (trans), 3.5 (cis) \\
\hline
\end{tabular}

amounts is a rather special situation for point defects. The existence of these clusters, demonstrated experimentally for $\mathrm{C}_{6}$ [7], should be attributed to an intermediate position of carbon between boron and nitrogen in the periodic table, as well as to the similar crystal structure of graphite and hBN.

(v) Among the defects that are neither single carbon substitutionals nor carbon clusters, a doubly negatively charged $\mathrm{C}_{\mathrm{B}} V_{\mathrm{B}}$ pair is the only defect that can reach concentrations of $10^{14}-10^{15} \mathrm{~cm}^{-3}$ in bulk samples grown under N-rich conditions. Our calculations show that these pairs do not have an internal optical transition. Thus they are unlikely to be SPEs observed in Ref. [13].

(vi) If oxygen is present during growth, it does not affect the concentration of carbon defects under $\mathrm{N}$-rich conditions. However, under $\mathrm{N}$-poor conditions, $\mathrm{C}_{\mathrm{N}} \mathrm{O}_{\mathrm{N}}$ defects are formed in densities exceeding those of any other carbon- or oxygencontaining defects by more than one order of magnitude. A $\mathrm{C}_{\mathrm{N}} \mathrm{O}_{\mathrm{N}}$ defect is expected to emit in the ultraviolet spectral region. It has been proposed as an alternative defect to the carbon dimer to explain the 4.1-eV luminescence [26].

(vii) All other carbon defects not discussed above occur at substantially smaller concentrations. This includes the $\mathrm{C}_{\mathrm{B}} V_{\mathrm{N}}$ defect, which was recently proposed to be the origin of single-photon emission in hBN [14] in the energy range of 1.6-2.1 eV. In this paper, we do not provide evidence for or against this attribution. It is firmly established that there exist several distinct classes of SPEs in $\mathrm{hBN}$, emitting in this energy range $[8,10,11]$. However, our work provides strong proof that $\mathrm{C}_{\mathrm{B}} V_{\mathrm{N}}$ cannot form in dense ensembles as observed in Ref. [13]. If $\mathrm{C}_{\mathrm{B}} V_{\mathrm{N}}$ indeed emits in the 1.6-2.1-eV energy range, thermodynamic modeling shows that there are emitters that occur in much larger concentrations.

(viii) Overall, our results show that it is possible that the carbon emitters observed in Ref. [13] are related to (i) carbon monomers, (ii) carbon trimers, or (iii) starlike defects. The conclusion about monomers reinforces the conclusion recently reached by Auburger and Gali [17]. The idea of carbon trimers as SPEs in the energy range 1.6-2.1 eV was first suggested by Jara et al. [15]. Lastly, starlike defects have been studied previously [47]; however, their optical signal was hitherto not discussed, and they have not yet been proposed as visible SPEs. These defects, at variance with other candidates, have a high-spin ground state and are thus interesting systems for further studies.

Table I summarizes our main findings regarding the most prominent carbon defects under both N-rich and N-poor conditions. Defect densities correspond to the situation without oxygen present.

Stepping aside from the relevance of our work to SPEs and looking at the materials chemistry of hBN more generally, our work indicates that the simultaneous presence of oxygen and carbon during growth has a particularly detrimental effect on the material quality, especially under $\mathrm{N}$-poor conditions. This is due to the very low formation energy of the $\mathrm{C}_{\mathrm{N}} \mathrm{O}_{\mathrm{N}}$ defect, which occurs in very large concentrations. If the presence of oxygen and carbon cannot be avoided, growth under more nitrogen-rich conditions is a pathway to improve the quality of hexagonal boron nitride.

Note added in proof. After the submission of this paper to the journal, two related works appeared on the arXiv preprint service. In Ref. [55], the authors investigated optical properties of several carbon clusters. In particular, accurate calculations of the ZPL energy of the $\mathrm{C}_{6}$ cluster were presented. The obtained energy of $4.21 \mathrm{eV}$ is in good agreement with our estimate of $4.0 \mathrm{eV}$ (Sec. V A). In Ref. [56], the authors analyzed the electronic structure of small carbon clusters, as well as complexes of carbon with vacancies, in terms of smaller constituent units that make up these defects. Whenever a direct comparison can be made, the results presented in that paper are in agreement with ours.

\section{ACKNOWLEDGMENTS}

We thank I. Aharonovich, E. Auksorius, and S. Lany for useful comments. This work has received funding from the European Social Fund (Project No. 09.3.3-LMT-K-712-230110) under a grant agreement with the Research Council of Lithuania. Computational resources were provided by the Interdisciplinary Center for Mathematical and Computational Modelling (ICM), University of Warsaw (Grant No. GB81-6), and the High Performance Computing center "HPC Sauletekis" in the Faculty of Physics, Vilnius University. 
[1] D. L. Duong, S. J. Yun, and Y. H. Lee, Van der Waals layered materials: Opportunities and challenges, ACS Nano 11, 11803 (2017).

[2] B. Gil, G. Cassabois, R. Cusco, G. Fugallo, and L. Artus, Boron nitride for excitonics, nano photonics, and quantum technologies, Nanophotonics 9, 3483 (2020).

[3] G. Cassabois, P. Valvin, and B. Gil, Hexagonal boron nitride is an indirect bandgap semiconductor, Nat. Photon. 10, 262 (2016).

[4] J. Sun, C. Lu, Y. Song, Q. Ji, X. Song, Q. Li, Y. Zhang, L. Zhang, J. Kong, and Z. Liu, Recent progress in the tailored growth of two-dimensional hexagonal boron nitride via chemical vapour deposition, Chem. Soc. Rev. 47, 4242 (2018).

[5] T. Taniguchi and K. Watanabe, Synthesis of high-purity boron nitride single crystals under high pressure by using $\mathrm{Ba}-\mathrm{BN}$ solvent, J. Cryst. Growth 303, 525 (2007).

[6] M. Onodera, K. Watanabe, M. Isayama, M. Arai, S. Masubuchi, R. Moriya, T. Taniguchi, and T. Machida, Carbon-rich domain in hexagonal boron nitride: Carrier mobility degradation and anomalous bending of the Landau fan diagram in adjacent graphene, Nano Lett. 19, 7282 (2019).

[7] O. L. Krivanek, M. F. Chisholm, V. Nicolosi, T. J. Pennycook, G. J. Corbin, N. Dellby, M. F. Murfitt, C. S. Own, Z. S. Szilagyi, M. P. Oxley, S. T. Pantelides, and S. J. Pennycook, Atomby-atom structural and chemical analysis by annular dark-field electron microscopy, Nature (London) 464, 571 (2010).

[8] T. T. Tran, K. Bray, M. J. Ford, M. Toth, and I. Aharonovich, Quantum emission from hexagonal boron nitride monolayers, Nat. Nanotechnol. 11, 37 (2016).

[9] R. Bourrellier, S. Meuret, A. Tararan, O. Stéphan, M. Kociak, L. H. Tizei, and A. Zobelli, Bright UV single photon emission at point defects in h-BN, Nano Lett. 16, 4317 (2016).

[10] G. Grosso, H. Moon, B. Lienhard, S. Ali, D. K. Efetov, M. M. Furchi, P. Jarillo-Herrero, M. J. Ford, I. Aharonovich, and D. Englund, Tunable and high-purity room temperature single-photon emission from atomic defects in hexagonal boron nitride, Nat. Commun. 8, 705 (2017).

[11] A. L. Exarhos, D. A. Hopper, R. R. Grote, A. Alkauskas, and L. C. Bassett, Optical signatures of quantum emitters in suspended hexagonal boron nitride, ACS Nano 11, 3328 (2017).

[12] A. Sajid, M. J. Ford, and J. R. Reimers, Single-photon emitters in hexagonal boron nitride: A review of progress, Rep. Prog. Phys. 83, 044501 (2020).

[13] N. Mendelson, D. Chugh, J. R. Reimers, T. S. Cheng, A. Gottscholl, H. Long, C. J. Mellor, A. Zettl, V. Dyakonov, P. H. Beton, S. V. Novikov, C. Jagadish, H. H. Tan, M. J. Ford, M. Toth, C. Bradac, and I. Aharonovich, Identifying carbon as the source of visible single-photon emission from hexagonal boron nitride, Nat. Mater. 20, 321 (2020).

[14] A. Sajid and K. S. Thygesen, VNCB defect as source of single photon emission from hexagonal boron nitride, 2D Mater. 7, 031007 (2020).

[15] C. Jara, T. Rauch, S. Botti, M. A. L. Marques, A. Norambuena, R. Coto, J. E. Castellanos-Águila, J. R. Maze, and F. Munoz, First-principles identification of single photon emitters based on carbon clusters in hexagonal boron nitride, J. Phys. Chem. A 125, 1325 (2021).

[16] K. Li, T. Smart, and Y. Ping, $\mathrm{C}_{2} \mathrm{C}_{\mathrm{N}}$ as a $2 \mathrm{eV}$ single-photon emitter candidate in hexagonal boron nitride, arXiv:2110.01787.
[17] P. Auburger and A. Gali, Towards ab initio identification of paramagnetic substitutional carbon defects in hexagonal boron nitride acting as quantum bits, Phys. Rev. B 104, 075410 (2021).

[18] K. Era, F. Minami, and T. Kuzuba, Fast luminescence from carbon-related defects of hexagonal boron nitride, J. Lumin. 24, 71 (1981).

[19] L. Museur, E. Feldbach, and A. Kanaev, Defect-related photoluminescence of hexagonal boron nitride, Phys. Rev. B 78 , 155204 (2008).

[20] M. Uddin, J. Li, J. Lin, and H. Jiang, Probing carbon impurities in hexagonal boron nitride epilayers, Appl. Phys. Lett. 110, 182107 (2017).

[21] T. Pelini, C. Elias, R. Page, L. Xue, S. Liu, J. Li, J. H. Edgar, A. Dréau, V. Jacques, P. Valvin, B. Gil, and G. Cassabois, Shallow and deep levels in carbon-doped hexagonal boron nitride crystals, Phys. Rev. Mater. 3, 094001 (2019).

[22] V. Solozhenko, A. Lazarenko, J.-P. Petitet, and A. Kanaev, Bandgap energy of graphite-like hexagonal boron nitride, J. Phys. Chem. Solids 62, 1331 (2001).

[23] A. Katzir, J. Suss, A. Zunger, and A. Halperin, Point defects in hexagonal boron nitride. I. EPR, thermoluminescence, and thermally-stimulated-current measurements, Phys. Rev. B 11, 2370 (1975).

[24] L. Weston, D. Wickramaratne, M. Mackoit, A. Alkauskas, and C. G. Van de Walle, Native point defects and impurities in hexagonal boron nitride, Phys. Rev. B 97, 214104 (2018).

[25] M. Mackoit-Sinkevičienè, M. Maciaszek, C. G. Van de Walle, and A. Alkauskas, Carbon dimer defect as a source of the $4.1 \mathrm{eV}$ luminescence in hexagonal boron nitride, Appl. Phys. Lett. 115, 212101 (2019).

[26] A. Vokhmintsev, I. Weinstein, and D. Zamyatin, Electronphonon interactions in subband excited photoluminescence of hexagonal boron nitride, J. Lumin. 208, 363 (2019).

[27] H. Hamdi, G. Thiering, Z. Bodrog, V. Ivády, and A. Gali, Stone-Wales defects in hexagonal boron nitride as ultraviolet emitters, npj Comput. Mater. 6, 178 (2020).

[28] A. Gottscholl, M. Kianinia, V. Soltamov, S. Orlinskii, G. Mamin, C. Bradac, C. Kasper, K. Krambrock, A. Sperlich, M. Toth, I. Aharonovich, and V. Dyakonov, Initialization and read-out of intrinsic spin defects in a van der Waals crystal at room temperature, Nat. Mater. 19, 540 (2020).

[29] A. Gottscholl, M. Diez, V. Soltamov, C. Kasper, A. Sperlich, M. Kianinia, C. Bradac, I. Aharonovich, and V. Dyakonov, Room temperature coherent control of spin defects in hexagonal boron nitride, Sci. Adv. 7, eabf3630 (2021).

[30] V. Ivády, G. Barcza, G. Thiering, S. Li, H. Hamdi, J.-P. Chou, Ö. Legeza, and A. Gali, Ab initio theory of the negatively charged boron vacancy qubit in hexagonal boron nitride, npj Comput. Mater. 6, 41 (2020).

[31] J. Heyd, G. E. Scuseria, and M. Ernzerhof, Hybrid functionals based on a screened Coulomb potential, J. Chem. Phys. 118, 8207 (2003).

[32] J. P. Perdew, K. Burke, and M. Ernzerhof, Generalized Gradient Approximation Made Simple, Phys. Rev. Lett. 77, 3865 (1996).

[33] P. E. Blöchl, Projector augmented-wave method, Phys. Rev. B 50, 17953 (1994).

[34] S. Grimme, Semiempirical GGA-type density functional constructed with a long-range dispersion correction, J. Comput. Chem. 27, 1787 (2006). 
[35] G. Kresse and J. Furthmüller, Efficient iterative schemes for ab initio total-energy calculations using a plane-wave basis set, Phys. Rev. B 54, 11169 (1996).

[36] I. Tomaszkiewicz, The enthalpy of formation of hexagonal boron nitride, Pol. J. Chem. 76, 891 (2002).

[37] Y. Gu, M. Zheng, Y. Liu, and Z. Xu, Low-temperature synthesis and growth of hexagonal boron-nitride in a lithium bromide melt, J. Am. Ceram. Soc. 90, 1589 (2007).

[38] S. B. Zhang and J. E. Northrup, Chemical Potential Dependence of Defect Formation Energies in GaAs: Application to Ga SelfDiffusion, Phys. Rev. Lett. 67, 2339 (1991).

[39] C. Freysoldt, B. Grabowski, T. Hickel, J. Neugebauer, G. Kresse, A. Janotti, and C. G. Van de Walle, First-principles calculations for point defects in solids, Rev. Mod. Phys. 86, 253 (2014).

[40] C. Freysoldt, J. Neugebauer, and C. G. Van de Walle, Fully Ab Initio Finite-Size Corrections for Charged-Defect Supercell Calculations, Phys. Rev. Lett. 102, 016402 (2009).

[41] D. B. Laks, C. G. Van de Walle, G. F. Neumark, P. E. Blöchl, and S. T. Pantelides, Native defects and self-compensation in ZnSe, Phys. Rev. B 45, 10965 (1992).

[42] S. Lany and A. Zunger, Dopability, Intrinsic Conductivity, and Nonstoichiometry of Transparent Conducting Oxides, Phys. Rev. Lett. 98, 045501 (2007).

[43] J. Bhang, H. Ma, D. Yim, G. Galli, and H. Seo, Firstprinciples predictions of out-of-plane group IV and $\mathrm{V}$ dimers as high-symmetry, high-spin defects in hexagonal boron nitride, ACS Appl. Mater. Interfaces 13, 45768 (2021).

[44] See Supplemental Material at http://link.aps.org/supplemental/ 10.1103/PhysRevMaterials.6.014005 for (i) formation energies of complexes of substitutional carbon with antisites and vacancies, (ii) formation energies of carbon pairs, and (iii) a discussion of the formation of carbon defects in the presence of a fixed amount of carbon atoms.

[45] B. Huang and H. Lee, Defect and impurity properties of hexagonal boron nitride: A first-principles calculation, Phys. Rev. B 86, 245406 (2012).
[46] N. Berseneva, A. Gulans, A. V. Krasheninnikov, and R. M. Nieminen, Electronic structure of boron nitride sheets doped with carbon from first-principles calculations, Phys. Rev. B 87, 035404 (2013).

[47] N. Berseneva, A. V. Krasheninnikov, and R. M. Nieminen, Mechanisms of Postsynthesis Doping of Boron Nitride Nanostructures with Carbon from First-Principles Simulations, Phys. Rev. Lett. 107, 035501 (2011).

[48] B. Huang and S.-H. Wei, Comment on "Mechanisms of Postsynthesis Doping of Boron Nitride Nanostructures with Carbon from First-Principles Simulations", Phys. Rev. Lett. 107, 239601 (2011).

[49] M. S. C. Mazzoni, R. W. Nunes, S. Azevedo, and H. Chacham, Electronic structure and energetics of $\mathrm{B}_{x} \mathrm{C}_{y} \mathrm{~N}_{z}$ layered structures, Phys. Rev. B 73, 073108 (2006).

[50] M. Winter, M. H. E. Bousquet, D. Jacquemin, I. Duchemin, and $X$. Blase, Photoluminescent properties of the carbon-dimer defect in hexagonal boron-nitride: A many-body finite-size cluster approach, Phys. Rev. Mater. 5, 095201 (2021).

[51] L. Muechler, D. I. Badrtdinov, A. Hampel, J. Cano, M. Rösner, and C. E. Dreyer, Quantum embedding methods for correlated excited states of point defects: Case studies and challenges, arXiv:2105.08705.

[52] R. O. Jones and O. Gunnarsson, The density functional formalism, its applications and prospects, Rev. Mod. Phys. 61, 689 (1989).

[53] T. Korona and M. Chojecki, Exploring point defects in hexagonal boron-nitrogen monolayers, Int. J. Quantum Chem. 119, e25925 (2019).

[54] Y. Wang and W. Windl, Native point defects from stoichiometry-linked chemical potentials in cubic boron arsenide, J. Appl. Phys. 129, 075703 (2021).

[55] S. Li, A. Pershin, G. Thiering, P. Udvarhelyi, and A. Gali, Ultraviolet quantum emitters in h-BN from carbon clusters, arXiv:2111.09428.

[56] P. Huang, M. Grzeszczyk, K. Vaklinova, K. Watanabe, T. Taniguchi, K. S. Novoselov, and M. Koperski, Carbon and vacancy centers in hexagonal boron nitride, arXiv:2112.14906. 